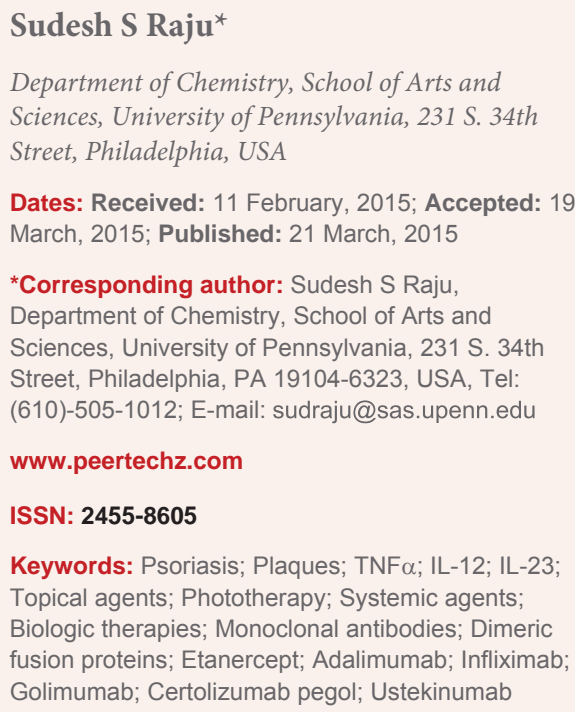

Sudesh S Raju*

Department of Chemistry, School of Arts and Sciences, University of Pennsylvania, 231 S. 34th Street, Philadelphia, USA

Dates: Received: 11 February, 2015; Accepted: 19 March, 2015; Published: 21 March, 2015

*Corresponding author: Sudesh S Raju,

Department of Chemistry, School of Arts and

Sciences, University of Pennsylvania, 231 S. 34th

Street, Philadelphia, PA 19104-6323, USA, Tel:

(610)-505-1012; E-mail: sudraju@sas.upenn.edu

www.peertechz.com

ISSN: 2455-8605

Keywords: Psoriasis; Plaques; TNF $\alpha$; IL-12; IL-23; Topical agents; Phototherapy; Systemic agents; Biologic therapies; Monoclonal antibodies; Dimeric fusion proteins; Etanercept; Adalimumab; Infliximab; Golimumab; Certolizumab pegol; Ustekinumab

Case Report

\section{Advances in Treatment Options for Psoriasis}

\section{Introduction}

Psoriasis is a complex autoimmune disorder that affects $\sim 2-3 \%$ of the human population [1]. Psoriasis has four major types: plaque, guttate, inverse, and pustular. Plaque psoriasis is the most prevalent and patients with plaque psoriasis suffer from red, itchy, scaly patches which can spread throughout the body [2]. Guttate psoriasis, occurring mostly in children, is seen as small pink-dots in the outer limbs [4]. Inverse psoriasis, commonly observed in overweight people, is evidenced by red lesion in the armpits and private regions [5]. Pustular psoriasis is accompanied by pustules (bumps filled with pus) that lead to fevers, nausea, and muscle weakness [6]. While psoriasis has many different sub-types, it is primarily due to epidermal hyper proliferation and inflammation. Although psoriasis is a recurring condition, it is not a fatal disease.

As a skin disease, psoriasis is mainly due to genetic predisposition and an environmental trigger. Within the genetic component, $\mathrm{T}$ cells and the major histocompatibility complex (MHC) play key roles in the progression of psoriatic inflammation [7]. Additionally, mutations within PSORS1 to PSORS9, IL12B of chromosome 5q and IL23R of chromosome 1p play critical roles within the inflammatory cascade $[7,10]$. Specifically, psoriatic patients have HLSA-Cw6 which codes for MHC class I protein and CDSM variant 5 , which leads to corneodesmosin being up-regulated within the epidermis of psoriatic patients [8]. From a genetic side, IL12B and IL23R are involved in the expression of their corresponding receptors, which together play critical roles in $\mathrm{T}$ cell differentiation and the inflammatory cascade with key molecules such as necrosis factor- $\alpha$ and nuclear factor $k B$ $[9,10]$. Additionally, patients suffering from guttate psoriasis have IL36Rn mutations.

Although genetic mutations are critical in engendering psoriasis, environmental triggers are often needed to induce an inflammatory response. Within an adaptive immune response, an antigen enters the body and prompts antigen-presenting skin cells (APSCs) to penetrate the lymphatic system, activate $\mathrm{T}$ cells, and engender the secretion of cytokines which induce the inflammatory cascade and psoriatic skin plaques $[11,12]$. However, in the innate immune system there are more identifiable mediators. For instance, toll-like receptors (TLRs) which are type 1 membrane glycoproteins that recognize pathogen associated molecular patterns (PAMPs), are important in the inflammatory cascade [13]. Within this cascade the keratinocyte barrier can be disturbed, NK lymphocytes can be activated, and natural killer receptors can be expressed. Interferon gamma (IFN $\gamma$ ), released by NK lymphocytes, and TNF $\alpha$, released by keratinocytes, work together to create inflammation, which is a physical characteristic of plaque psoriasis [14,15]. Another prevalent characteristic of plaque psoriasis is epidermal hyperplasia. Within this condition, keratinocytes proliferate and within the focal regions Type 1 effectors accumulate and induce cytokines such as IL-12, IL23, IFN $\gamma$, and TNFa $[16,17]$. Specifically, IL-12 and IL-23 are related to $\mathrm{T}$ cell differentiation and activation of $\mathrm{T}$ helper 1 cells phenotypes.

\section{Signaling molecules and role of TNFa}

Additional cytokines play critical roles in adhesion, sub-type specific influx, trafficking and compartmentalization of leukocytes [18]. These include TARC and MDC, which preferentially recruit skin-homing memory $\mathrm{T}$ cells via the stimulation of integrin-ICAM-1 adhesive interactions [19]. T cells and Langerhans skin cells also express chemokine receptor GPR-1 (CCR10) for the ligand CTACK (CCL27), which significantly contributes to epidermal localization. Keratinocytes can also induce MIP-3a, which is another proinflammatory cytokine [20]. In addition, the epidermis accumulates neutrophils (Munro's microabscesses), which are extremely prevalent in psoriasis [21]. Within the psoriatic scales, there is high content of IL-8 and GRO- $\alpha$, which are neutrophil-attracting chemokines [21]. IL-8 down regulates IL-10 receptor such that there is a loss of immune-regulation within psoriatic lesions.

TNFa is a pleiotropic pro-inflammatory cytokine that is markedly up-regulated in psoriatic patients. It is produced by activated 
macrophages, natural killer (NK) cells, $\mathrm{CD} 4^{+}$and $\mathrm{CD} 8^{+} \mathrm{T}$ cells in response to stimulation by antigens, pathogens, immune complexes, pro-inflammatory cytokines and TNFa itself by auto-feedback loop [22]. TNFa is a transmembrane homo-trimeric protein, with each monomer being $\sim 26 \mathrm{kDa}$ in molecular weight [23]. Upon proteolytic cleavage by TACE/ADAM17, a soluble trimer of TNFa of $\sim 51 \mathrm{kDa}$ is released as an inflammatory cytokine [23]. The soluble version of TNFa binds to TNF receptor 1 (TNFR1; p55 CD120a) and TNF receptor 2 (TNFR2; p75; CD120b) [24]. Both TNF receptors 1 and 2 are part of a family of membrane and soluble proteins that are involved in cell differentiation, survival, apoptosis, production of cytokines and chemokines and the inflammatory response [25]. Specifically, TNFR1 is the primary receptor of TNFa activity, while TNFR2 is involved in the activation of $\mathrm{T}$ cells, regulation of lymphocytes, and synergization with TNFR1 [25].

In addition, TNFa, which stimulates the production of chemokines, is a chemo-attractant protein that mediates the stimulation and activation of lymphocytes [26]. TNFa up-regulates adhesion molecules and then facilitates lymphocytes to the skin. It also increases the vascular endothelial growth factor, which increases capillary permeability in angiogenesis [26]. This increase in capillary density, recruitment of immune cells to the epidermis, and accumulation of keratinocytes and other mediators within the inflammatory cascade, all lead to erythema and psoriatic plaques [26]. Additionally, TNFa induces macrophages to produce cytokines IL- 6 and IL-1 [28]. TNFa also increases expression of adhesion molecules [24].

Also, the TNF receptors, upon binding to TNFa, undergo conformational changes in which SODD (silencer of death domains) is released from the intracellular domain [24]. This allows for the TRADD (TNFa receptor 1 associated death domain protein) to bind to the death domain and allow for activation of NF-kB, which is a transcription factor that plays a critical role in inflammation, activation of MAPK pathways, and induction of death signaling [24]. Hence, targeting cytokines, chemokines, TNF- $\alpha$, and interleukins are effective therapeutic goals in treating plaque psoriasis.

\section{Treatment options}

Currently there are a wide range of therapies available to combat plaque psoriasis. These include topical treatments, phototherapy, tonsillectomy, systemic therapies and biologics. Topical treatments and systemic therapies are being prescribed as the first line treatment options whereas phototherapy is no longer in use because of significant side effects. Recently, advances have been made to understand the molecular basis of psoriasis which led to the development of new class of biologics as treatment options.

\section{Topical treatment}

The most common medication is topical treatment, a medication that is applied directly to skin plaques. These include corticosteroids, anthracene derivatives, vitamin D3 derivatives, and retinoids. These topical agents work by normalizing excessive cell proliferation, reducing inflammation, and hindering swelling to prevent psoriatic plaques [27]. Some of the topical steroids are derived from the corticosteroid hormones that are produced by the human adrenal glands. Other topical agents incorporate salicylic acid, which is a keratolytic (peeling agent), to directly remove the scales of psoriatic plaques [28]. Dovonex (calcipotriene) uses synthetic vitamin D3 to hinder cell growth and to remove lesions. Taclonex (calcipotriene and betamethasone dipropionate) slows epidermal cell growth and reduces inflammation [29]. Tazorec (tazarotene) is a vitamin A derivative, vectical (calcitriol) is a natural form of Vitamin D3, and zithranol-RR (antralin) uses synthetic chrysarobin [29]. Although topical agents are the first-line of defense for mild to moderate psoriasis, other treatment options also exist.

\section{Phototherapy}

Another common type of treatment for psoriasis is phototherapy, which is a method that has been in place since 1976. Phototherapy is more commonly known as Ultra-violet therapy and it is used to treat moderate to severe plaque psoriasis. The methodology relies on using UV-C (100-290nm), UV-B (290-320nm), and UV-A (320$400 \mathrm{~nm}$ ) light that lead to keratinocyte apoptosis and hence reduce the development of plaques within the affected individuals [30]. However, this therapy has many limits because the exact mechanism is yet to be understood and repeated UV exposure is known to lead to skin cancer [30].

\section{Tonsillectomy}

Streptococcal infection has been connected to the onset of plaque psoriasis within a subset of patients. A critical component within this triggering mechanism could be the infection activating $\mathrm{T}$ cells to epitopes of keratin within the skin [31]. Since, palatine tonsils are an immunological organ that may create auto-reactive $\mathrm{T}$ cells to epitopes within the skin, tonsillectomy has recently gained traction as a valuable treatment option for psoriatic patients [31].

\section{Systemic therapies}

Systemic therapies are treatment options that affect systems involving the entire body. One well known type of systemic drug is methotrexate, which was found to be an effective treatment for psoriasis since 1950s and was approved by the FDA in the 1970s [32]. Psoriatic patients can take methotrexate orally in either as a pill or liquid form once a week [32]. On the molecular level, methotrexate is a class of medications known as antimetabolites that inhibit enzymes within purine metabolism, inhibit $\mathrm{T}$ cell activation, and suppress intercellular adhesion molecule expression by $\mathrm{T}$ cells [33]. Since psoriasis has a large inflammatory component that is mediated by activated T cells and adhesion molecules, methotrexate works well as a systemic agent to target molecules within the inflammatory cascade to prevent the progression of psoriasis.

Other forms of systemic therapies, such as cyclosporine and acitretin (Soriatane), work well against plaque psoriasis but also carry significant side effects. Specifically, cyclosporine daily, is an oral drug that combats psoriasis by suppressing the growth of immune and skin cells [34]. Hence, long-term use of cyclosporine compromises the immune system and has side effects such as impaired kidneys and high blood pressure [35]. Acitretin, which is developed from vitamin A, is a daily oral drug that works well with phototherapy to combat psoriasis [36]. Specifically, acitretin affects the growth and shedding 
process of skins cells; however, it has numerous side effects such as hair loss, depression, and birth defects [37].

\section{Biologics}

Recently many biological drugs have been developed to treat several autoimmune diseases including psoriasis. Biological drugs are mainly recombinant and/or purified therapeutic proteins that are derived either from living cells cultured in laboratory settings or purified from living sources [38]. These drugs are given by injection or intravenous (IV) infusion [38]. While biological drugs purified from living sources such as human plasma, have been successfully used to treat human diseases for the past several decades, modern molecular biologic and biochemical techniques have greatly accelerated the production of effective biological drugs using recombinant methods [38]. Unlike systemic drugs which are known to impact the entire immune system non-specifically, biological drugs have the potential to work more effectively and specifically [38]. These biological drugs have the ability to target specific molecules within the immune system [38]. The most prevalent biological drugs developed to treat psoriasis target specifically either TNF $\alpha$ or interleukins 12 and 23 [38]. Since these molecules play specific roles during the development of plaque psoriasis, targeting them with biological molecules has been very effective. Currently available biological therapies to treat psoriasis are monoclonal antibodies targeting either TNFa or interleukins 12 and 23, and an IgG1 fusion protein targeting TNFa.

Monoclonal antibodies and fusion proteins as specific biological therapies for psoriasis

Monoclonal antibodies (MAbs) are mono-specific antibodies that are made by the same immune cells, in contrast to the polyclonal antibodies that are made by different immune cells [39]. MAbs are particularly useful in that they bind to a particular epitope and hence are specific for molecules [39]. MAbs were previously made by hybridoma cells in which mice were immunized by a particular antigen and then myeloma cells were fused with the spleen cells from the mice to produce a cell line that would constitutively produce antibodies [39]. However, since MAbs were made from mice and not humans, there existed notable structural differences [40]. Hence, another approach was used by adopting recombinant DNA method to produce chimeric antibodies in that they are part mouse and part human.

In contrast, dimeric IgG fusion proteins consists of polypeptides that each have non-immunoglobin polypeptide domains and a dimerizing IgG Fc domain along with a polypeptide linker [41]. Dimeric IgG fusion proteins are particularly important in increasing the serum half-life of polypeptide chains and hence are very useful in treating the inflammatory cascade for plaque psoriasis [41].

The advances made in producing MAbs and dimeric IgG fusion proteins shown that biological therapies are effective options for treating plaque psoriasis [42]. The anti-TNF MAbs binds to TNFa and the anti-IL12 and IL23 MAb binds to the p40 shared domain of IL-12 and IL23 [42]. Upon binding, these biological therapies can control the progression of plaque psoriasis by inhibiting specific signaling molecules within the inflammatory cascade. These biological therapies include Enbrel, Humira, Remicade, Simponi, and
Cimzia. Additionally, there are several more in the advanced phase of clinical trials.

Amgen is marketing Enbrel (etanercept), which was approved by USFDA to treat moderate to severe psoriasis [43]. Enbrel is an IgG fusion protein in which the Fc region of human IgG1 is fused to two domains of TNFa receptor 2 (p75) [43]. The TNFa receptor 2 domain allows Enbrel to bind to TNFa, the $\mathrm{F}_{c}$ region IgG1 part of Enbrel allows for it to bind to $\mathrm{B}$ cells, and the mast cell $\mathrm{F}_{\mathrm{c}}$ receptors allow for reduced stimulation [43]. Enbrel has a molecular weight of $\sim 110 \mathrm{kDa}$ and binds to TNF $\alpha$ with $11 \mathrm{pM}$ affinity [44]. Psoriatic patients are treated with Enbrel with monthly injections [44]. Additionally, since Enbrel is a fusion protein it has an extended serum half-life within the blood stream and has long lasting biological effects [44].

Humira (adalimumab) is a fully human anti-TNFa MAb based therapeutic drug developed by Abbott Laboratories and has been approved by USFDA to treat plaque psoriasis [45]. Humira's molecular weight is $\sim 148 \mathrm{kDa}$ and it contains human IgG1 kappa chain framework. Humira binds specifically to the soluble and transmembrane bound TNFa [45]. Hence Humira effectively inhibits TNFa function by steric interaction. Humira has 14 days of in vivo half-life within the serum and binds to soluble TNFa with $\sim 127 \mathrm{pM}$ affinity [46]. Psoriatic patients take $\sim 160 \mathrm{mg}$ loading dose, $80 \mathrm{mg}$ second dose after two weeks, and $40 \mathrm{mg}$ dose every two weeks thereafter as injections directly under the skin [47]. Humira is another effective biological treatment for psoriatic patients as it inhibits both the soluble and transmembrane form of TNFa, which is a key component in the inflammatory cascade found within psoriatic plaques.

Remicade (infliximab) is a mouse-human chimeric anti-TNFa MAb based drug marketed by Janssen Biotech to treat psoriasis and psoriatic arthritis [48]. It is a $\sim 149 \mathrm{kDa}$ protein that has IgG1 kappa chain that binds specifically to soluble TNFa and transmembrane $\mathrm{TNF} \alpha$ to inhibit the pro-inflammatory functions of TNFa [48]. Remicade binds to TNFa with $\sim 44 \mathrm{pM}$ affinity [49]. In addition, Remicade has an in vivo half-life of roughly 8.5 days [49]. Psoriatic patients take Remicade through IV; with $4 \mathrm{mg} / \mathrm{kg}$ patient at initial weeks and then 2 to $6 \mathrm{mg} / \mathrm{kg}$ every 8 weeks thereafter [49]. Remicade is also an effective biological treatment for psoriatic patients due to its ability to specifically inhibit TNFa, which is a pro-inflammatory cytokine found up-regulated within psoriatic patients.

Simponi (golimumab) is a fully human IgG1 kappa MAb, produced by Janseen Biotech that also binds to soluble TNF $\alpha$ as well as the transmembrane bound TNF $\alpha$ to inhibit the pro-inflammatory functions of TNF $\alpha$ [50]. Simponi has a molecular weight of $\sim 147 \mathrm{kDa}$, in vivo half-life of 14 days, and binds to soluble TNFa with $18 \mathrm{pM}$. Psoriatic patients take Simponi once a month in $50 \mathrm{mg}$ dose as injections directly under the skin [51]. Simponi binds directly to TNFa and inhibits its pro-inflammatory functions [51].

Cimzia (certolizumab pegol) PEG is a human IgG1 kappa chain Fab' fragment conjugated to PEG and has an approximate molecular weight of $\sim 95 \mathrm{kDa}$ [52]. It is also a biological drug that binds to both soluble and transmembrane TNFa. Cimzia blocks the proinflammatory functions of TNFa. It has $\sim 14$ days of in vivo human 
serum half-life and it binds to soluble TNF $\alpha$ with $\sim 90$ pM affinity [52]. Psoriatic patients take $400 \mathrm{mg}$ of Cimzia via injection at week 0,2 , and 4 , and then $200 \mathrm{mg}$ every other week thereafter [52].

Other than TNFa inhibitors, one more biological drug currently available to treat psoriasis is Stelara (ustekinumab). Stelara is a fully human IgG1k MAb marketed by Janseen Biotech and targets both interleukin-12 and interleukin-23 [53]. Stelara functions as an effective biological therapy for psoriatic patients by binding to the common p40 subunit of interleukin-12 and interleukin-23 [v]. These two cytokines are involved in the activation of $\mathrm{T}$ cells and are key mediators in the Th1 and Th17 inflammatory pathways [53]. Psoriatic patients take $45 \mathrm{mg}$ injections of Stelara at weeks 0 and 4, and then every 3 months thereafter [53]. Stelara works by blocking IL-12 and IL-23 from activating $\mathrm{T}$ cells, which play a crucial component within the inflammatory cascade of the development of plaque psoriasis; hence, it is an effective treatment option for psoriatic patients.

While the aforementioned biological drugs are effective in treating moderate to severe plaque psoriasis, there are side effects, especially in relation to the monoclonal antibodies and dimeric fusion proteins. For instance, many of the biological drugs suppress the immune system, hence they increase the chances of infections and some forms of cancer [54]. Other side effects include headaches, abdominal pain, and upper-respiratory infections [54].

Currently, there are numerous biological drugs in the research and development pipeline to treat psoriasis. For example, Amgen and AstraZeneca completed phase III trials of Brodalumab to treat moderate to severe arthritis. Brodalumab works by binding to the IL-17 receptor and blocking IL-17 receptor ligands from inducing inflammation [55]. Brodalumab is taken by subcutaneous injection every two weeks at a dosage of either $140 \mathrm{mg}$ or $210 \mathrm{mg}$ [55]. Brodalumab is one of many other monoclonal antibodies that show promise in treating plaque psoriasis.

\section{Conclusion}

While plaque psoriasis is a recurring disease many treatment options exist such as topicals, phototherapy, and systemic agents. Well-known and new biological therapies have shown much potential for significantly curbing the progression of the disease. Since cytokines such as TNFa, IL-12, and Il-23 play significant roles in the progression of the inflammatory cascade, biological drugs have been developed to target these cytokines and hence reduce the development and advancement of plaque psoriasis. Anti-TNFa MAbs such as etanercept, adalimumab, infliximab, and golimumab bind to soluble TNFa and inhibit its role in inflammation. Additionally, ustekinumab targets IL-12 and IL-23, which then delays the activation of $\mathrm{T}$ cells, which also play critical roles in inflammation. Hence, current biological therapies and future ones that will target additional cytokines within the inflammatory cascade have the potential to make plaque psoriasis a much more manageable disease.

\section{Expert Commentary}

Psoriasis is a chronic autoimmune disease that has many different risk factors including genetic predisposition and environmental triggers. Psoriatic patients often use topical agents such as those that incorporate corticosteroids as a first-line defense to control the epidermal hyper proliferation. Phototherapy is another common option as it induces keratinocyte apoptosis. Additionally, systemic agents are effective in treating psoriasis but have many side effects mainly because they are non-specific and have impacts throughout the body. Recent advances in the study of psoriasis and other autoimmune diseases have shown that mediators within the inflammatory cascade can be specifically inhibited by biological drugs with minimal side effects. Currently, there is a plethora of antiTNF $\alpha$ mAbs on the market, with many binding within the picomolar range, and effectively inhibiting the progression of inflammation and development of plaque psoriasis. Other biological drugs such as ustekinumab inhibit interleukins that coordinate $\mathrm{T}$ cell activation, which are critical mediators of inflammation as well.

\section{Five-year review}

Although significant advances have been made in the past 30 years in the study of psoriasis and similar autoimmune diseases, additional research in the next five years will allow for a greater understanding of unresolved mediators within the inflammatory cascade to more comprehensively treat psoriatic patients. Recently much emphasis has been placed on the role of Th1, Th2, and Th17 and their associated cytokines in the initiation and advancement of psoriasis. Current and future experimental studies will be able to better discern the roles of each of these helper $\mathrm{T}$ cells lineages in differentiation, cytokine production, and progression of psoriasis.

\section{Key Issues}

- Psoriasis currently affects $2-3 \%$ of the human population.

- Psoriasis has many forms such as guttate, inverse, and pustular; however, plaque psoriasis is the most common and it is notable for inflammation.

- Genetic predisoposition within the genes for T cell and major histocompatibility complex is strongly correlated with the onset and progression of plaque psoriasis.

- An environmental trigger is introduced which leads to an adaptive and innate immune response.

- An inflammatory cascade results and manifests into plaque psoriasis.

- Signaling molecules such as TNFa, IL-12, and IL-23 play critical roles within the inflammatory cascade and the manifestation of plaque psoriasis.

- Therapies include topical, UV, systemic agents, and biological drugs.

- Anti-TNFa mAbs have been proven as successful and new biologic drugs such as dimeric fusion proteins to target interleukins show much promise in treating and controlling plaque psoriasis.

\section{Financial and Competing Interest Disclosure}

This author is affiliated with the Department of Chemistry at the University of Pennsylvania. The author has no relevant affiliations or competing financial interests with any organizations with a financial interest or conflict with the topic or materials described within this 
manuscript. No assistance was used in writing of this manuscript.

\section{References}

1. Langley RG, Krueger GG, Griffiths CE (2005) Psoriasis: Epidemiology, Clinical Features, and Quality of Life. Ann Rheum Dis 64: 18-23.

2. Myers WA, Gottlieb AB, Mease P (2006) Psoriasis and Psoriatic Arthritis: Clinical Features and Disease Mechanisms. Clin Dermatol 24: 438-447.

3. Naldi L, Gambini D (2007) The Clinical Spectrum of Psoriasis. Clinics in Dermatology 25: 510-518.

4. Kwon HH, Na SJ, Jo SJ (2011) Epidemiology and Clinical Features of Pediatric Psoriasis in Tertiary Referral Psoriasis Clinic. J Dermatol 39: $260-$ 264.

5. Syed Z, Khachemoune A (2011) Inverse Psoriasis: Case Presentation and Review. Am J Clin Dermatol 12: 143-146.

6. Choon S, Lai N, Mohammad N (2014) Clinical Profile, Morbidity, and outcome of adult-onset generalized pustularpsoriasis: Analysis of 102 Cases Seen in a Tertiary Hospital in Johor, Malaysia. IntJ Dermatol 53: 676-684.

7. Bowcock AM, Krueger JG (2005) Getting under the Skin: The Immunogenetics of Psoriasis. Nat Rev Immunol 5: 699-711.

8. Gudjonsson JE, Karason A, Antonsdottir A (2003) Psoriasis Patients Who Are Homozygous for the HLA-Cw*0602 Allele Have a 2.5-fold Increased Risk of Developing Psoriasis Compared with Cw6 Heterozygotes. $\mathrm{Br} \mathrm{J}$ Dermatol 148: $233-235$

9. Becher B, Pantelyushin S (2012) Hiding under the Skin: Interleukin-17producing $ү \delta T$ Cells Go under the Skin? Nat Med18: 1748-1750.

10. Tracey D, Klareskog L, Sasso E (2008) Tumor Necrosis Factor Antagonis Mechanisms of Action: A Comprehensive Review. Pharmacol Ther1 17: 244279 .

11. Capon F (2013) IL36RN Mutations in Generalized Pustular Psoriasis: Just the Tip of the Iceberg? J Invest Dermatol 133: 2503-2504.

12. Griffiths CE, Richards HL (2001) Psychological Influences in Psoriasis. Clini Exp Dermatol 26: 338-342.

13. Arnetz BB, Fjellner B, Eneroth $P$ (1985) Stress and psoriasis: psychoendocrine and metabolic reactions in psoriatic patients during standardized stressor exposure. Psychosom Med 47: 528-541.

14. Medzhitov R, Janeway CA Jr (1997) Innate Immunity: Impact on the Adaptive Immune Response. Curr OpinImmuno I 9: 4-9.

15. Connolly D, O'Neill L (2012) New Developments in Toll-like Receptor Targeted Therapeutics. Curr Opin Pharmacol 12: 510-518.

16. Victor FC, Gottlieb AB, Menter A (2003) Changing Paradigms in Dermatology: Tumor Necrosis Factor Alpha (tnf- $\alpha$ ) Blockade in Psoriasis and Psoriatic Arthritis. Clin Dermatol 21: 392-397.

17. Matsushima K (1989) Interleukin 8 and MCAF: Novel Inflammatory Cytokines Inducible by IL 1 and TNF. Cytokine 1: 2-13.

18. Austin LM, Ozawa M, Kikuchi T (1999) The Majority of Epidermal T Cells in Psoriasis Vulgaris Lesions Can Produce Type 1 Cytokines, Interferon- $\gamma$, Interleukin-2, and Tumor Necrosis Factor- $\alpha$, Defining TC1 (Cytotoxic T Lymphocyte) and TH1 Effector Populations:1 a Type 1 Differentiation Bias Is Also Measured in Circulating Blood T Cells in Psoriatic Patients. J Invest Dermatol 113: 752-759.

19. Campbell JJ, Haraldsen G, Pan J (1999) The chemokine receptor CCR4 in vascular recognition by cutaneous cells but not intestinal memory $T$ cells. Nature 400: 776-780.

20. Homey B, Dieu-Nosjean MC, Wiesenborn A (2000) Up-regulation of macrophage inflammatory protein-3 alpha/CCL20 and CC chemokine receptor 6 in psoriasis. J Immunol 164: 6621-6632.

21. Pease JE (2006) Asthma, Allergy and Chemokines. Curr Drug Targets 7: 3-12.
22. Tracey D, Klareskog L, Sasso EH (2008) Tumor necrosis factor antagonist mechanisms of action: a comprehensive review. Pharmacol Ther 117: 244279.

23. Horiuchi T, Mitoma H, Harashima S (2010) Transmembrane TNFa: structure, function and interaction with anti-TNFa agents. Rheumatology 49: 12151228.

24. Zelova H, Hosek J (2013) TNFa signaling and inflammation: interactions between old acquaintances. Inflamm Research 62: 641-651.

25. Croft M (2009) The role of TNF superfamily members in T-cell function and diseases. Nat Rev Immunol9: 271-285

26. Nograles KE, Davidovici B, Krueger JG (2010) New Insights in the Immunologic Basis of Psoriasis. Sem Cutan Med Surg 29: 3-9.

27. Papoutsaki M, Costanzo A (2013) Treatment of psoriasis and psoriatic arthritis. Bio Drugs27: 3-12.

28. Famenini S, Wu JJ (2013) Combination therapy with tumor necrosis factor inhibitors in psoriasis treatment. Cutis 92: 140-147.

29. Segaert S, Ropke M (2013) The biological rationale for use of vitamin d analogs in combination with corticosteroids for the topical treatment of plaque psoriasis. J Drugs Dermatol 12: e129-137.

30. Weatherhead, Sophie, Peter Farr, Nicholas Reynolds (2013) Spectral Effects of UV on Psoriasis. Photochem Photobiol Sci 12: 47-54.

31. Wu V, Debbaneh M, Moslehi H, Koo J, Liao W (2014) Tonsillectomy as a treatment for psoriasis" a review. J Dermatol Treat 25: 482-486.

32. Mease P (2013) Methotrexate in psoriatic arthritis. Bull Hosp Jt Dis 71: 41-45.

33. Tamilselvi E, Haripriya D, Hemamalini M (2013) Association of Disease Severity with IL-1 levels in Methotrexate-treated Psoriasis Patients. Scad J Immunol 78: 545-553.

34. Kelly JB III, Foley P, Strober BE (2015) Current and future oral systemic therapies for psoriasis. Dermatol Clin 33: 91-109.

35. Shaw MK, Davis SA, Feldman SR (2014) Trends in systemic psoriasis treatment therapies form 1993 through 2010. J Drugs Dermatol 13: 917-920.

36. Schaarschmidt ML, Kromer C, Herr R (2014) Treatment Satisfaction of Patients with Psoriasis. Act DermVenereol [Epub ahead of print]

37. Mahmood T, Zaghi D, Menter A (2015) Emerging oral drugs for psoriasis. Expert OpinEmerg Drugs 3:1-12.

38. Crow JM (2012) Therapeutics: Silencing Psoriasis. Nature 492: 58-59.

39. Lin J, Ziring D, Desai S (2008) TNFa Blockade in Human Diseases: An Overview of Efficacy and Safety. Clinical Immunology 126: 13-30.

40. Chaudhari U, Romano P, Mulcahy L (2001) Efficacy and Safety of Infliximab Monotherapy for Plaque-type Psoriasis: A Randomised Trial. Lancet 357: 1842-1847

41. Boado RJ, Hui EK, Lu JZ (2013) IgG-enzyme fusion protein: pharmacokinetics and anti-drug antibody response. Bioconjug Chem 24: 97-104.

42. Semble AL, Davis SA, Feldman SR (2014) Safety and tolerability of tumor necrosis factor- $\alpha$ inhibitors in psoriasis: anarrative review. Am J Clin Dermatol 15: $37-43$

43. van den Reek JM, van Lumig PP, Driessen RJ (2014) Determinants of drugsurvival of etanercept for psoriasis in a long-term daily practice cohort. $\mathrm{Br} \mathrm{J}$ Dermatol170: 415-424.

44. Neovius M, Arkema EV, Olsson H (2015) Drug survival on TNF inhibitors in patients with rheumatoid arthritis comparison of adalimumab, etanercept and infliximab. Ann Rheum Dis 74: 354-360.

45. Puig L (2014) Biologic Therapies for Moderate to Severe Psoriasis are not Interchangeable. Actas Dermosifiliogr 105: 483-486.

46. Hsu L, Snodgrass BT, Armstrong AW (2013) Anti-drug Antibodies in Psoriasis: A systematic Review. Br J Dermatol 170: 261-273. 
47. Hsu L, Armstrong AW (2013) Anti-drug antibodies in psoriasis: a critical evaluation of clinical significance and impact on treatment response. Expert Rev Clinlmmunol 9: 949-958.

48. Papoutsaki M, Osorio F, Morais P (2013) Infliximab in psoriasis and psoriatic arthritis. Bio Drugs 1: 13-23.

49. Arsiwala S (2013) Infliximab: efficacy in psoriasis. Indian J Dermatol Venereol Leprol 7: S25-34.

50. Hayashi T (2013)Golimumab. Nihon Rinsho 7: 1227-1231.

51. Chimenti MS, Teoli M, Saracena R (2013) Golimumab in patients affected by moderate to severe psoriatic arthritis: an open-label study in thirty-two patients previously treated with other biologics. Dermatology 227: 305-310.
52. Mease PJ, Fleischmann R, Deodhar AA (2014) Effect of certolizumabpegol on signs and symptoms in patients with psoriatic arthritis: 24-week results of a Phase 3 double-blind randomised placebo-controlled study (RAPID-PsA) Ann Rheum Dis 73: 48-55.

53. Gottlieb A, Narang K (2013)Ustekinumab in the treatment of psoriatic arthritis: latest findings and clinical potential. Ther Adv Musculoskelet Dis 5: 277-285

54. Jensen JD, Delcambre MR, Nguyen G (2014) Biologic therapy with or without topical treatment in psoriasis: what does the current evidence say? Am J Clin Dermatol. 15: 379-395.

55. Papp K, Leonardi C, Menter A (2014) Safety and efficacy of brodalumab for psoriasis after 120 weeks of treatment. J Am Acad Dermatol 71: 1183-1190.

Copyright: (C) 2015 Raju SS. This is an open-access article distributed under the terms of the Creative Commons Attribution License, which permits unrestricted use, distribution, and reproduction in any medium, provided the original author and source are credited. 